\title{
Promoting Hand Sanitizer Use in a University Cafeteria
}

\section{Christoph Bördlein ${ }^{1}$}

Published online: 10 February 2020

(C) The Author(s) 2020

\begin{abstract}
Handwashing is the most cost-efficient method to lower the risk of the transmission of infectious diseases. Especially before eating in public places like cafeterias, handwashing is recommended. Often, people do not wash their hands before eating because of the response effort associated with going to the bathroom. As an alternative way to improve hygiene, disinfection with a hand sanitizer gel is recommended. The current study used an A-B-C-A design (and a no-intervention control site) with prompts and feedback to increase the number of cafeteria patrons using hand sanitizer. Dispensers for hand sanitizer gel were placed at the entrance area of two halls of a university cafeteria. Intervention took place in one hall, whereas the other served as a control. After baseline, a poster explaining the usefulness of hand sanitizing was posted near the entrance of the cafeteria. This led to a doubling of the percentage of cafeteria patrons using the hand sanitizer, from $10.79 \%$ during baseline to $24.45 \%$. A second poster provided feedback about the percentage of patrons sanitizing their hands and asked for more participation. This led to no further increases in hand sanitizing (23.73\%). After all posters were removed, the percentage dropped to $15.63 \%$ in the mean. This research demonstrated that a simple, informative prompt can have a considerable impact on hand-sanitizing behavior. However, the role of feedback in such interventions remains questionable.
\end{abstract}

Keywords Behavioral community intervention $\cdot$ Feedback $\cdot$ Hand hygiene $\cdot$ Hand sanitizer gels $\cdot$ Hand sanitizing $\cdot$ Prompts

Hand hygiene is essential for the prevention of transmittable diseases (SaundersHastings, Crispo, Sikora, \& Krewski, 2017; Wong, Cowling, \& Aiello, 2014). Regular handwashing with nonantibacterial plain soap can reduce respiratory infections and

Christoph Bördlein

christoph.boerdlein@fhws.de

1 Faculty of Applied Social Science, University of Applied Sciences Würzburg-Schweinfurt, Münzstr. 12, 97070 Würzburg, Germany 
gastrointestinal illness by $31 \%$ and $21 \%$, respectively (Aiello, Coulborn, Perez, \& Larson, 2008) and is considered sufficient as a preventive measure outside patient care. Alternatively, alcohol-based solutions are effective in preventing diseases (Picheansathian, 2004).

Health authorities recommend regularly washing hands or using hand sanitizer gel after using restrooms, blowing one's nose, returning home, and before eating or treating wounds (Centers for Disease Control, 2017). But even among professional health care workers, hand hygiene occurs far too infrequently (Ho, Ansari, \& Page, 2014), especially when there is no control of compliance (Wu et al., 2018). Improving hand hygiene in the general public would improve public health, but interventions to improve hand hygiene outside the health care sector are sparse, and the results are inconclusive (see Schweizer et al., 2014; Willmott et al., 2016). Education, reminders, and feedback have been used, but there is no decisive evidence for a lasting effect of any of these interventions.

Behavior-analytic interventions in this field are often based on prompts and feedback (e.g., Choi, Lee, Moon, \& Oah, 2018). From other areas of application, it is known that prompts are often less effective than feedback in changing behavior (e.g., Moon \& Oah, 2013). However, in these studies, the feedback is specific and individualized. The group receiving the feedback is either small or even $N=1$. Feedback works best when it is directed at the behavior of a person or a small group of people in close relationship to each other (see Alvero, Bucklin, \& Austin, 2001). But interventions to improve hand hygiene behavior in the public sphere do not address a specific group of people. People using a restroom or cafeteria patrons are not a well-defined group; group membership changes over time, as some people use the facilities only one time during an intervention. This situation is characteristic of behavioral community interventions (Luyben, 2009). In behavioral community interventions, the target behavior (e.g., someone washing or sanitizing his or her hands) is emitted not by certain identifiable individual subjects, but rather by people united only through an accidental commonality like using the same restroom (see Bördlein, 2018). Behavioral community interventions often use prompts and feedback to modify the behavior of people in the public sphere. If the feedback is delivered repeatedly, the recipients of the feedback may change over time, as it is not always the same people using the restroom. Therefore, the feedback used in these interventions probably does not function as a behavioral consequence at the individual level, but rather as an antecedent (see Duncan $\&$ Bruwelheide, 1985, and Peterson, 1982, for discussions on the behavioral function of feedback).

Interventions using feedback to increase hand hygiene behaviors are sparse. One recent example is Choi et al. (2018). The authors compared the effects of visual prompts and feedback on the handwashing behavior of restroom users. The prompt was a sign that stated "Washing hands with soap can prevent the transmission of various diseases"; the feedback was another sign, stating the percentage of persons washing their hands on the previous day. Choi et al. (2018) found that the feedback intervention was more effective than the prompt intervention. However, the feedback also told the people using the restroom that the handwashing behavior of restroom users was surveilled by an observer, standing in proximity to the sink and pretending to use his smartphone. This added a social consequence to the situation, which suggests that it is possible that negative reinforcement was at work during the feedback intervention. 
People washed their hands during the feedback interventions to avoid the embarrassment of being observed and recorded as someone leaving the restroom without washing his or her hands by an observer standing nearby. It remains questionable whether feedback is more effective than prompts in behavioral community interventions aimed at the improvement of hand hygiene behaviors, especially when the feedback does not imply a component of social surveillance and possible negative reinforcement. Therefore, the question as to whether feedback is effective in interventions aimed at hand hygiene behaviors is still open. The current research addresses this question with a feedback intervention component that did not imply that the individual behavior was personally surveyed, as was the case in the study by Choi et al. (2018). This, however, would allow only feedback directed at the behavior of a larger group of people, not specific and individualized feedback such as that advised in the literature on feedback (Alvero et al., 2001, p. 20).

Behavioral interventions should be based on an analysis of the contingencies of the behavior targeted. Functional analyses have revealed that it is often the response effort connected with hand hygiene behaviors that keeps people from performing the required measures (Casella et al., 2010). Therefore, the response effort required for the behavior should be kept as small as possible.

Fournier and Berry (2012) observed students entering a university cafeteria to determine whether they used the restroom to wash their hands or used a hand sanitizer gel before going to the meal delivery location. Although informational posters and change agents were effective in increasing the rate of students using the hand sanitizer gel, they were ineffective with respect to washing hands in the restroom. The authors assumed that the differential response effort of using the hand sanitizer gel and going to the restroom is critical in promoting hand hygiene.

In the current study, hand sanitizer gel dispensers were placed at the entrance of a university cafeteria. A prompt and a feedback intervention were tested for their effect on the rate of cafeteria patrons using the hand sanitizer gel before going to the meal delivery location. The aim of the study was to compare the effects of an informative prompt alone with a prompt and nonpunitive feedback, which simply informed the cafeteria patrons about the percentage of patrons sanitizing their hands and asked them to sanitize their hands too.

\section{Method}

\section{Participants and Setting}

This study was conducted in the university cafeteria of a German university. The cafeteria was subdivided into two dining halls, a bigger one (Hall 1) and a smaller one (Hall 2). Students and employees of two universities nearby used the cafeteria for lunch. According to the operating agency of the cafeteria (student services), during the period of the study (May 3 to June 20), an average of 907.6 meals per day was served during lunchtime (from 11:30 a.m. to 2:00 p.m.) in Hall 1; in Hall 2, it was 672.64 meals per day. In both halls, immediately after the entrance of the hall, was a small area where the cafeteria patrons could pick up their silverware together with a dinner tray. Then the cafeteria patrons proceeded to the meal delivery location. 
Before the study began, student services provided three hand sanitizer gel dispensers. Each dispenser was mounted on a stand that was $150 \mathrm{~cm}$ high in total. The dispenser itself was $30 \mathrm{~cm}$ high. Inside the dispenser, a bottle with an antibacterial sanitizer gel, containing 1 liter of fluid, was inserted. The dispenser operated contactfree. When someone placed his or her hand approximately 3-5 cm under the dispenser, approximately $3 \mathrm{ml}$ of fluid were automatically released by the dispenser in the cupped hand of the person.

Two dispensers were placed in the area between the entrance and the silverware counter of Hall 1, on the left and the right sides. Another dispenser was placed in the entrance area of Hall 2. The intervention took place only in Hall 1. Hall 2 served as a control site, where only the consumption of hand sanitizer gel was measured. No behavior observations took place in Hall 2.

\section{Dependent Variable, Measurement, and Interobserver Agreement}

The dependent variable was the percentage of cafeteria patrons using the hand sanitizer gel. Using the hand sanitizer gel was defined as a person, after entering the cafeteria, holding one or both of his or her hands under the dispenser, whereupon sanitizer gel was released. This made an audible sound and could be seen from a distance of $2 \mathrm{~m}$. Subsequently, the person rubbed his or her hands for at least $5 \mathrm{~s}$. Health authorities and producers of hand sanitizer gel recommend using the gel for at least $30 \mathrm{~s}$ (Aktion Saubere Hände, 2015). But informal observations revealed that no cafeteria patron in fact used the gel for more than 10-15 s.

The dependent variable was recorded by two observers. One observer, pretending to read and write, sat at a table in a gallery above the entrance area of Hall 1, where he could observe the cafeteria entrance. Each person entering the cafeteria by stepping over the doorsill was counted as a cafeteria patron. A second observer stood in the entrance area of Hall 1 and pretended to use her smartphone. The second observer recorded the number of cafeteria patrons using the hand sanitizer gel. The rate of cafeteria patrons using the hand sanitizer gel was calculated by dividing the number of hand sanitizer users recorded by Observer 2 by the number of cafeteria patrons recorded by Observer 1, and multiplying by 100 .

Observations took place from Monday to Thursday from 12:00 p.m. to 12:30 p.m., when the attendance of the cafeteria was greatest. On average, 321 patrons entered Hall 1 during this period (range 261-363). The observations began on May 3 and ended on June 20. During $25 \%$ of all observation sessions, two independent observers were present for the calculation of interobserver agreement (IOA), using frequency-withininterval recording. The observation period of $30 \mathrm{~min}$ was subdivided into intervals of 5 min. IOA for the number of cafeteria patrons was $99.61 \%$ (range $98.83 \%-100 \%$ ), and IOA for the number of cafeteria patrons using hand sanitizer gel was $98.78 \%$ (range $96.83 \%-100 \%)$.

As a second measurement in the form of product recording, the usage of hand sanitizer gel in both halls was recorded. The smaller dining hall, Hall 2, was used as a control site. Before starting observations of hand-sanitizing behavior, the observers opened the dispensers and marked with a permanent marker the level of sanitizer in the bottle. If the bottle was empty or nearly empty (less than 5\% content remaining), observers changed it. Unfortunately, the bottles deformed while they emptied; 
therefore, it was not possible to use the level markers as a reliable measure of the usage of sanitizer gel. Nevertheless, the number of bottles used in Hall 1 and Hall 2 during baseline and intervention could be used as an indicator of the usage of hand sanitizer gel (see Results).

After the intervention phases, an online survey about the social validity of the intervention was conducted. The author advertised the online survey in social media groups for local students. Participants were asked whether they frequented the cafeteria regularly and if they found it important to sanitize their hands before eating. They were also asked if they appreciated the hand sanitizer gel dispensers in the cafeteria, if they had noticed the posters in the cafeteria that encouraged cafeteria patrons to use the hand sanitizer gel, if they liked the posters, and if they thought that the posters had an influence on their behavior of sanitizing their hands. All items had to be answered on a 5-point Likert-type scale from 1 (I agree completely.) to 5 (I disagree completely.). The survey was online for 14 days, beginning at the end of the second intervention phase.

\section{Experimental Design and Procedures}

An A-B-C-A design with prompts and feedback and a no-intervention control site was used. After baseline, a prompt intervention was followed by a prompt plus feedback intervention and a return to baseline.

Baseline The study started with the setup of the sanitizer gel dispensers. The dispensers had a display on top with a panel of approximately $13 \mathrm{~cm} \times 10 \mathrm{~cm}$, stating "Hand Sanitizer." During the following six sessions, the baseline observations took place.

Prompt The first intervention component (prompt) was a poster, $841 \mathrm{~mm} \times$ $1,189 \mathrm{~mm}$ in size. The poster stated how many germs a person would pick up when touching a toilet flush handle, a door latch, and a smartphone display. This was illustrated by photos of the items, together with comic characters symbolizing the germs. A fourth photo showed a cutlery tray with comic characters symbolizing the germs. The poster stated "After lecture, before the lunch, sanitize your hands!" The poster was placed on the wall of the gallery crossing the entrance area of Hall 1 , approximately $3.5 \mathrm{~m}$ above the ground. It was clearly visible when patrons entered the cafeteria.

Prompt and feedback After six other observation sessions, a second poster was placed (the first poster remained in place). This poster was $420 \mathrm{~mm} \times 594 \mathrm{~mm}$ in size and hung directly next to one of the sanitizer gel dispensers in the entrance area of Hall 1 . The poster showed bar graphs of the daily percentage of cafeteria patrons using hand sanitizer gel during the last 8 days and an "Uncle Sam" comic character that stated "We want you for disinfection." The bar graphs were updated immediately after each observation session.

Return to baseline After six additional intervention sessions, the posters were removed, and the return to baseline was conducted. 


\section{Results}

\section{Percentage of Patrons Using Hand Sanitizer}

Figure 1 shows the percentage of cafeteria patrons who used hand sanitizer gel after entering the cafeteria. During baseline, relatively low percentages were observed $(M=$ $10.79 \%)$. When the prompt was introduced, average percentages immediately increased to more than double $(M=24.45 \%)$ the baseline phase percentage. After the introduction of the second poster (feedback plus prompt phase), the average percentage remained at the same level as during the prompt phase $(M=23.73 \%)$. The removal of the two posters, during return to baseline, brought an immediate decline in the average percentage to a level slightly above the first baseline $(M=15.63 \%)$.

\section{Hand Sanitizer Gel Use}

As an additional measurement, the usage of hand sanitizer gel in Hall 1 (intervention site) and Hall 2 (control site) was recorded. Unfortunately, it was not possible to take a more precise measurement than the number of sanitizer gel bottles used. During the first baseline, one bottle of sanitizer gel was used in Hall 2 (with only one dispenser), and two bottles were used in Hall 1 (with two dispensers). During the intervention phases, whereas eight bottles were used in Hall 1, in Hall 2 usage remained at baseline level with two bottles used. In the second baseline phase, less than two bottles were used in Hall 1 and less than one bottle in Hall 2.

\section{Social Validity}

Seventy-nine participants filled out the online survey (11 male, 66 female, 2 other or missing); $34 \%$ frequented the cafeteria every day or nearly every day. Participants found it generally important to wash or sanitize their hands before eating $\left(M=1.93, s^{2}\right.$ $=0.57)$. They approved of the dispensers set up in the cafeteria $\left(M=1.61, s^{2}=1.14\right)$. The posters were noticed $\left(M=2.84, s^{2}=1.48\right)$ and found somewhat appealing $(M=$ $2.62, s^{2}=1.12$ ). However, the participants tended not to believe that the posters had an influence on their decision to use the hand sanitizer gel $\left(M=3.17, s^{2}=1.32\right)$.

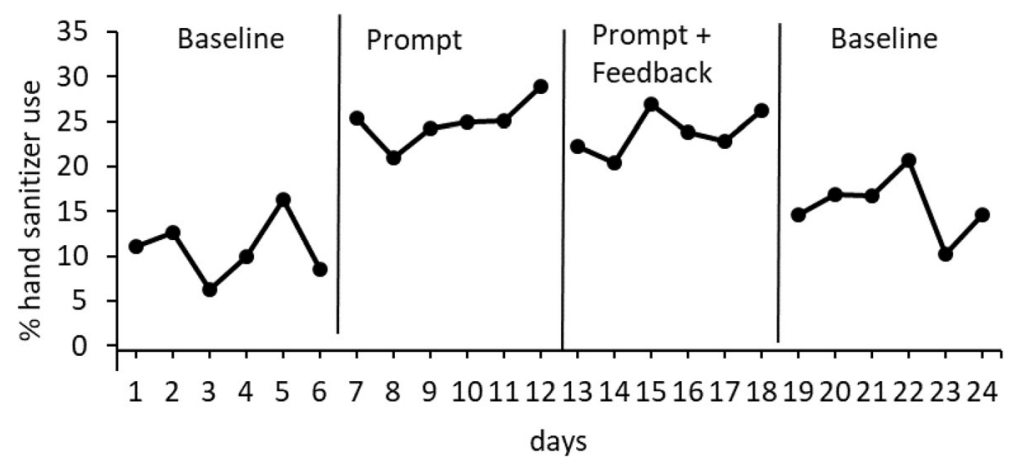

Fig. 1. Percentage of hand sanitizer gel use 


\section{Discussion}

The current study compared a prompt intervention with a prompt and feedback intervention aimed at improving hand hygiene of cafeteria patrons. A prompt poster was somewhat effective in promoting the use of hand sanitizer gel in a university cafeteria. A feedback poster, posted in addition to the prompt poster, resulted in no further increases in the rate of cafeteria patrons using hand sanitizer gel. Rates of hand sanitizing dropped to a level slightly above preintervention levels after the posters were removed. The evidence for the effectiveness of the intervention is further strengthened by the higher usage of sanitizer gel in the intervention site (Hall 1) compared to the control site (Hall 2). Although the behavior of the cafeteria patrons was clearly influenced by the prompt poster, the participants in the online survey denied that they changed their behavior because of the posters. However, the effect on the population of cafeteria patrons was limited. Approximately $75 \%$ of patrons were not affected by the interventions. The effects of the intervention were not maintained after the intervention ended.

A possible reason for the ineffectiveness of the feedback poster was the nature of the feedback. The feedback referred to the behavior of a large group of people, but group membership was not stable over the observation sessions. Only one third of the cafeteria patrons used the cafeteria regularly. Although the behavioral function of feedback is debated (Mangiapanello \& Hemmes, 2015; Peterson, 1982), it is known that feedback works better when it is related to individual behavior (Alvero et al., 2001). If the person receiving the feedback cannot relate it to his or her own behavior (because he or she has not been to the cafeteria during the previous days), a feedback graphic of the percentage of patrons using hand sanitizer gel functions only as a behavioral antecedent, not as a consequence for individual behavior. Cafeteria patrons who see the feedback only once are not influenced much in their behavior. A longer exposure to feedback could have resulted in increases in the rate of hand sanitizer use, as more cafeteria patrons would have seen the feedback several times. In a related field (energy consumption), it has been shown that feedback works best when it is given frequently and over a long period (Fischer, 2008).

A limitation of the current study was that the sequence of the interventions was not counterbalanced. However, because the dispensers were placed in the cafeteria only before the baseline sessions took place, it was considered sensible to start with the prompt intervention to inform cafeteria patrons about the opportunity for hand sanitizing. Due to time restraints, another baseline phase between the two intervention phases (prompt only, prompt plus feedback) was not possible. Also, the study could have ended with an intervention phase (prompt only, as this was the effective component). But experimental control was demonstrated even with the current design.

Another limitation is the operational definition of hand sanitizing, which required the person to rub the gel on their hands for only a few seconds. Hand sanitizer gel is maximally effective when used for at least $30 \mathrm{~s}$. However, no cafeteria patron used the hand sanitizer gel for so long. A subsequent intervention could aim at increasing the residence time of the gel, to maximize the effects of hand sanitizer gel use on public hygiene.

The operator of the cafeteria decided to keep the hand sanitizer gel dispensers and to advertise hand sanitizing by posting informational posters at the cafeteria entrance. A 
control of the effectiveness by recording the amount of sanitizer gel used was recommended. If the usage of hand sanitizer gel drops, additional interventions to increase the rates of hand sanitizing are advisable.

Future studies should aim at increasing the effectiveness of feedback. Feedback should be individualized and combined with reinforcers to be maximally effective. An automated system that gives feedback every time the dispenser is used is conceivable. For example, a tablet computer, attached to the dispenser, would enable the delivery of individualized feedback. Every time the dispenser is used, an observer would activate the tablet and a message like "Thank you for sanitizing your hands" would appear on the screen. However, this feedback will only function as a behavioral consequence if it is used over a longer period, so patrons repeatedly contact the feedback.

This study adds to the existing literature on the influence of prompts and feedback on hand hygiene behaviors. Certain prompts increase hand-sanitizing behavior. However, the effect of feedback components in such settings should be investigated further.

Author Note The author is grateful to Veronika Horlebein, Hanna Zwingmann, Kerstin Njeri, Johanna Memhardt, Anna-Maria Göbel, Bernhard Seibold, Sarah Tozman, and Katrin Salzinger for their contribution to the research and data collection. The author would like to thank Raymond Miltenberger for his comments on an earlier draft of this article.

Funding Information Open Access funding provided by Projekt DEAL.

\section{Compliance with Ethical Standards}

Conflict of Interest The author declares no conflict of interest.

Ethical Approval The author's university does not have an ethical committee, and the author did not find it necessary to have the study approved by ethical committees outside the university. However, the study has been performed in accordance with the ethical standards as laid down in the 1964 Declaration of Helsinki and its later amendments or comparable ethical standards.

Informed Consent Informed consent was obtained from all individual participants who filled out the social validity questionnaire.

Open Access This article is licensed under a Creative Commons Attribution 4.0 International License, which permits use, sharing, adaptation, distribution and reproduction in any medium or format, as long as you give appropriate credit to the original author(s) and the source, provide a link to the Creative Commons licence, and indicate if changes were made. The images or other third party material in this article are included in the article's Creative Commons licence, unless indicated otherwise in a credit line to the material. If material is not included in the article's Creative Commons licence and your intended use is not permitted by statutory regulation or exceeds the permitted use, you will need to obtain permission directly from the copyright holder. To view a copy of this licence, visit http://creativecommons.org/licenses/by/4.0/.

\section{References}

Aiello, A. E., Coulborn, R. M., Perez, V., \& Larson, E. L. (2008). Effect of hand hygiene on infectious disease risk in the community setting: A meta-analysis. American Journal of Public Health, 98, 1372-1381. https://doi.org/10.2105/AJPH.2007.124610. 
Aktion Saubere Hände. (2015). Händedesinfektion: Fakten und Informationen [Hand sanitation: Facts and information]. Retrieved from http://www.aktion-sauberehaende.de/ash/patienten-undangehoerige/haendesdesinfektion/

Alvero, A. M., Bucklin, B. R., \& Austin, J. (2001). An objective review of the effectiveness and essential characteristics of performance feedback in organizational settings (1985-1998). Journal of Organizational Behavior Management, 21(1), 3-29. https://doi.org/10.1300/J075v21n01_02.

Bördlein, C. (2018). Student-directed behavioral community interventions in social work education. Research on Social Work Practice, 28, 731-741. https://doi.org/10.1177/1049731518755011.

Casella, S. E., Wilder, D. A., Neidert, P., Rey, C., Compton, M., \& Chong, I. (2010). The effects of response effort on safe performance by therapists at an autism treatment facility. Journal of Applied Behavior Analysis, 43, 729-734. https://doi.org/10.1901/jaba.2010.43-729.

Centers for Disease Control. (2017). Hand washing: Clean hands save lives. Retrieved from https://www.cdc. gov/handwashing/index.html

Choi, B., Lee, K., Moon, K., \& Oah, S. (2018). A comparison of prompts and feedback for promoting handwashing in university restrooms. Journal of Applied Behavior Analysis, 51, 667-674. https://doi. org/10.1002/jaba.467.

Duncan, P. K., \& Bruwelheide, L. R. (1985). Feedback: Use and possible behavioral functions. Journal of Organizational Behavior Management, 7(3-4), 91-114. https://doi.org/10.1300/J075v07n03_06.

Fischer, C. (2008). Feedback on household electricity consumption: A tool for saving energy? Energy Efficiency, 1, 79-104. https://doi.org/10.1007/s12053-008-9009-7.

Fournier, A. K., \& Berry, T. D. (2012). Effects of response cost and socially-assisted interventions on handhygiene behavior of university students. Behavior and Social Issues, 21, 152-164. https://doi.org/10.5210 /bsi.v21i0.3979.

Ho, J. D., Ansari, R. K., \& Page, D. (2014). Hand sanitization rates in an urban emergency medical services system. The Journal of Emergency Medicine, 47, 163-168. https://doi.org/10.1016/j. jemermed.2013.08.070.

Luyben, P. D. (2009). Applied behavior analysis: Understanding and changing behavior in the community-a representative review. Journal of Prevention \& Intervention in the Community, 37, 230-253. https://doi. org/10.1080/10852350902975884.

Mangiapanello, K. A., \& Hemmes, N. S. (2015). An analysis of feedback from a behavior analytic perspective. The Behavior Analyst, 38, 51-75. https://doi.org/10.1007/s40614-014-0026-x.

Moon, K., \& Oah, S. (2013). A comparison of the effects of feedback and prompts on safe sitting posture: Utilizing an automated observation and feedback system. Journal of Organizational Behavior Management, 33, 152-162. https://doi.org/10.1080/01608061.2013.785906.

Peterson, N. (1982). Feedback is not a new principle of behavior. The Behavior Analyst, 5, 101-102. https://doi.org/10.1007/BF03393144.

Picheansathian, W. (2004). Effectiveness of alcohol-based solutions for hand hygiene: A systematic review. JBI Library of Systematic Reviews, 2(9), 1-27. https://doi.org/10.11124/jbisrir-2004-375.

Saunders-Hastings, P., Crispo, J. A. G., Sikora, L., \& Krewski, D. (2017). Effectiveness of personal protective measures in reducing pandemic influenza transmission: A systematic review and meta-analysis. Epidemics, 20, 1-20. https://doi.org/10.1016/j.epidem.2017.04.003.

Schweizer, M. L., Reisinger, H. S., Ohl, M., Formanek, M. B., Blevins, A., Ward, M. A., \& Perencevich, E. N. (2014). Searching for an optimal hand hygiene bundle: A meta-analysis. Clinical Infectious Diseases, 58, 248-259. https://doi.org/10.1093/cid/cit670.

Willmott, M., Nicholson, A., Busse, H., MacArthur, G. J., Brookes, S., \& Campbell, R. (2016). Effectiveness of hand hygiene interventions in reducing illness absence among children in educational settings: A systematic review and meta-analysis. Archives of Disease in Childhood, 101, 42-50. https://doi. org/10.1136/archdischild-2015-308875.

Wong, V. W. Y., Cowling, B. J., \& Aiello, A. E. (2014). Hand hygiene and risk of influenza virus infections in the community: A systematic review and meta-analysis. Epidemiology and Infection, 142, 922-932. https://doi.org/10.1017/S095026881400003X.

Wu, K.-S., Lee, S. S.-J., Chen, J.-K., Chen, Y.-S., Tsai, H.-C., Chen, Y.-J., et al. (2018). Identifying heterogeneity in the Hawthorne effect on hand hygiene observation: A cohort study of overtly and covertly observed results. BMC Infectious Diseases, 18, 369. https://doi.org/10.1186/s12879-018-3292-5. 